\title{
Towards a Visual Analytics Framework for Handling Complex Business Processes
}

\author{
William Ribarsky \\ Department of Computer Science \\ University of North Carolina at Charlotte \\ 9201 University City Blvd, Charlotte, NC, 28262 \\ ribarsky@uncc.edu
}

\author{
Wenwen Dou \\ Department of Computer Science \\ University of North Carolina at Charlotte \\ wdou1@uncc.edu
}

\author{
Derek Xiaoyu Wang \\ Department of Computer Science \\ University of North Carolina at Charlotte \\ xiaoyu.wang@uncc.edu
}

\author{
William J. Tolone \\ Department of Software and Information Systems \\ University of North Carolina at Charlotte \\ William.Tolone@uncc.edu
}

\begin{abstract}
Organizing data that can come from anywhere in the complex business process in a variety of types is a challenging task. To tackle the challenge, we introduce the concepts of virtual sensors and process events. In addition, a visual interface is presented in this paper to aid deploying the virtual sensors and analyzing process events information. The virtual sensors permit collection from the streams of data at any point in the process and transmission of the data in a form ready to be analyzed by the central analytics engine. Process events provide a uniform expression of data of different types in a form that can be automatically prioritized and that is readily meaningful to the users. Through the visual interface, the user can place the virtual sensors, interact with and group the process events, and delve into the details of the process at any point. The visual interface provides a multiview investigative environment for sensemaking and decisive action by the user.
\end{abstract}

\section{Introduction}

The idea of "just in time (JIT) manufacturing" has been around for some time and has been implemented in large scale production environments. Lately there has been the need for dynamic just in time manufacturing where the distribution and even types of products produced may change fairly quickly, often in response to a previously unforeseen need. This is the case for some large government entities that must supply materiel in support of changing missions and for some large manufacturers.
In this paper we describe a framework we have developed in cooperation with a partner engaged in dynamic manufacturing. A major issue is that the business process (in this case manufacture and delivery of a variety of systems, which could be complex themselves or components of even larger systems) is complex and changing. It can be across several parts of a large manufacturing and delivery organization and across many different suppliers. A disruption at any point in the organization or among the suppliers could result in a failure to deliver full capabilities on time, which would be a serious problem.

A main issue that can arise is shown in the following example, illustrated in Figure 1. Suppose the assembly of a system that must be deployed by a certain date is dependent on the availability of a component that is provided by an external supplier. Further the component is not installed till late in the assembly process. Since this is a dynamic manufacturing process where different systems with different externally supplied components are required to be produced at different times, the organizational structure cannot be closely aligned with the manufacturing process. In this case the procurement and assembly components are in different part of the organizational structure with different reporting paths. In the illustration, a clue to the problem is given by unusual communication being opened up between the parts acquisition/preparation department and the quality assurance manager. (This isn't the only possible path; the communication between the production supervisor and the technical manager could possibly be due to the same or a related problem.) There may also be unusual activity within a department even without external communication. In Figure 1, this is picked up by a "virtual sensor" (dis- 


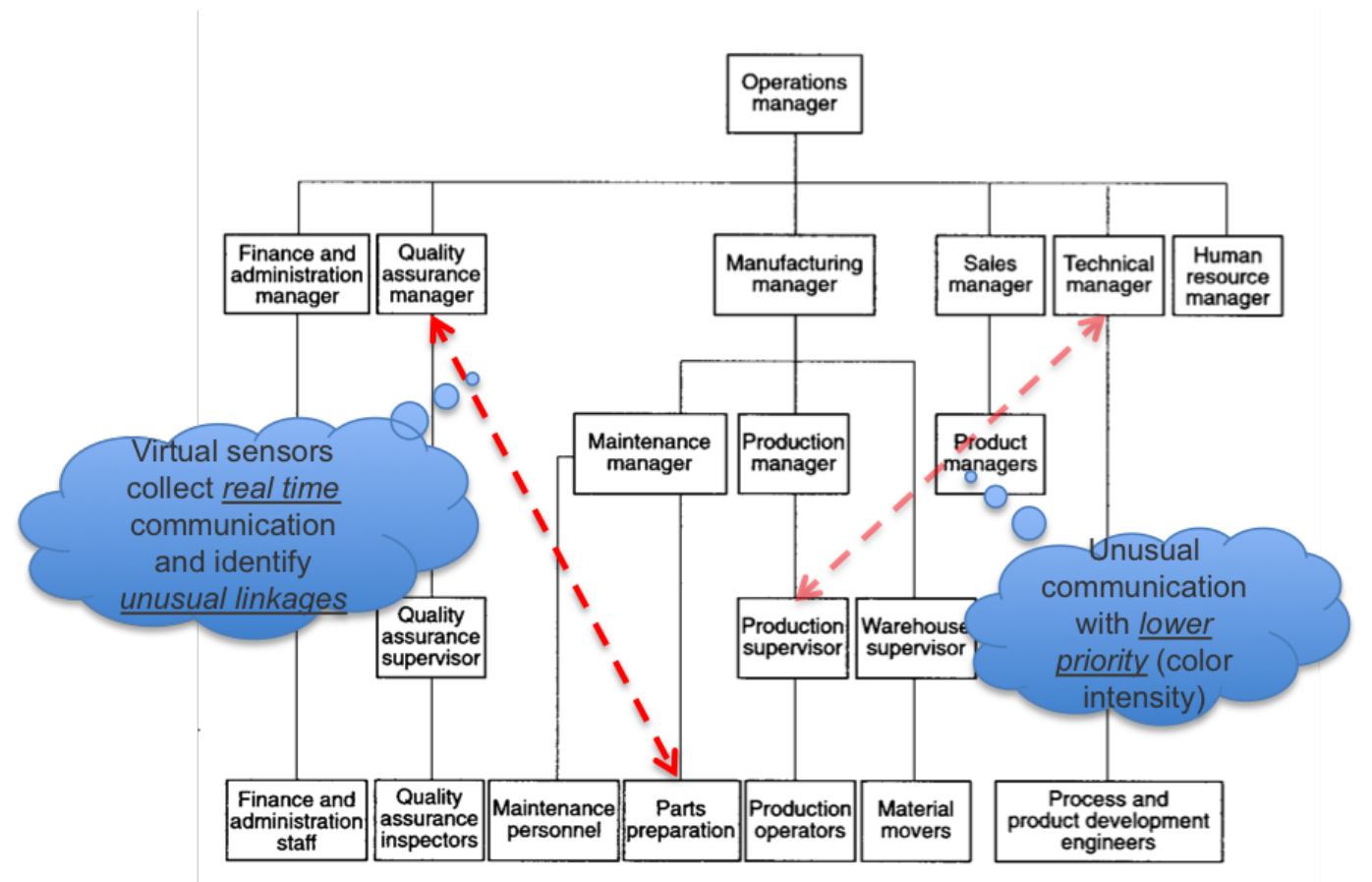

Figure 1. An organization structure for a production operation with unusual communication paths.

cussed further below and in Sec. 5.) Unless there is appropriate and timely communication across the structure and in particular to the operations manager (and above), the manufacturing process will break down. This is to be contrasted with a non-dynamic JIT process. In this case, the steps of the process are more fixed, permitting better organizational alignment from the start and better communication. Furthermore, non-dynamic JIT permits predictive planning where a forecast model based on past production and other factors is imposed to predict inventory needs, among other needs [15]. Such predictive methods will be much harder to develop in dynamic JIT manufacturing. Finally, most JIT planning approaches have been applied to much more uniform manufacturing processes (e.g., the production of a certain car model) than are the case here, where quite different systems are produced at different times.

The main contribution of this paper is the development of a framework to attack the above problem by applying a visual analytics approach. Although there has been work in process-aware information systems (see Related Work), these approaches haven't been applied to manufacturing processes nor, more importantly, are they as comprehensively data-driven as our approach. To enable our approach, we have introduced two new concepts: virtual sensors and process events. Virtual sensors are software collector/transmitters that can be placed at will anywhere in the data flow of the manufacturing or production process. They are meant to deal with a range of data from quantitative process information to communications among operators or managers. Since the virtual sensors monitor the stream of data over time, the visual analytics framework can identify process events, meaningful occurrences in time with respect to the process. We have shown how general event-based approaches can organize complex data histories in a semiautomatic way $[14,19]$. What this means is that the visual analytics analysis can reveal the data process and through it the underlying business process. If, for example, the data process does not conform with the organizational model of the manufacturing business, this can signal problems that should be addressed.

\section{Related Work}

There is a history of business process visualization in the business industry $[3,13,16,20]$. In the area of customer relations management, Azvine et al. presented a configurable business process analytics tool that constantly monitors the performance of a decision model on both overall and individual levels with the goals of customer satisfaction and operational excellence [1]. Noting that existing business analytic applications are usually closed-loop decision making systems which only present output to operational managers, Azvine et al. incorporated visualization components into an intelligent business analytics system in order to put users in the loop and adjust business operations based on users' 
analyses in real time.

In the domain of Process-Aware Information System (PAIS), which pertains to both administrative processes and cross-organizational processes, visualization techniques have been used to aid in the understanding of process schemas and their run-time behavior through simulation [7] and process mining [11]. For example, to improve business process models, Alast et al. combined process mining with visual analytics to incorporate human judgment into otherwise static business process models [11]. On the one hand, process mining supports automatic discovery of a business model and checking how a process model conforms to actual process executions. On the other hand, visual analytics combines automated analysis with interactive visualizations to allow decision-makers to apply their flexibility, creativity, and domain knowledge to come to an effective understanding of situations in the context of large data sets. The authors noted that insights obtained from visualizations could be used to improve processes by removing inefficiencies and addressing non-compliance. In addition to process mining, visualization techniques have also been applied to visualizing work items for an overall business process [12]. Leoni et al. incorporated visualizations to support work assignment in process-aware information systems. The visualizations enable users to better select work items to ensure the performance of the overall business process [12].

\section{Probing Dynamic Business Processes}

In collaboration with our partner, we have developed the following set of questions that must be effectively addressed in order to achieve a successful dynamic business process:

- What are overall trends? Is the overall process on schedule?

- What are the detailed trends for individual programs within the manufacturing and production process? (A program is the production of a particular system, usually distinct from other systems that may also be produced during the production process.)

- What?s the cost-schedule performance over time?

- What is the capacity of each part of the operation, including excess capacity?

- Is something going wrong and where (including things that have not been fully recognized yet)?

- Is resource re-allocation needed, and where?

- If there is a problem, what expertise should be deployed to most quickly solve it?
To have any chance of answering these questions, the production executives must have continuously available the latest information on the manufacturing process at the program and component levels. To this end, our partner has developed a "dashboard" that accepts data from all stages of the process for all programs. The dashboard system has both an interface and a data collector that provides a summary analysis to determine where the process, at any stage, stands with respect to a few simple benchmarks. Importantly, production managers can provide annotations and comments at any point in the process. They can also communicate with each other or the executives via the dashboard. The dashboard displays comparative information on the production process for all components and programs. It shows some information on trends and when a component is falling behind schedule according to a simple milestone analysis. It has some drill-down capability so that the executive can get more information about why a trend is occurring.

Although this sort of analysis is necessary, our partner has found it is not sufficient for enabling a dynamic business process without interruptions or missed deadlines. In particular, the dashboard does not have a way to effectively organize and use all the information it collects, especially the annotations and comments that are critical to understanding the production process. In addition, disruptions in different parts of the organization may affect downstream production in unexpected ways. Further, there may be no direct line of communication between the parts of the organization that will be affected (although there could be informal communication). Neither of these aspects are readily apparent to the manager in the dashboard setup. Finally, since unexpected disruptions will arise, it is only possible to know of them after the fact and often with incomplete information as to cause. Thus it is difficult to put into place predictive or even prospective monitoring and response, nor to pursue some of the questions above such as those having to do with re-allocating resources and expertise as an event is unfolding. In the next sections, we discuss how visual analytics can be applied to address these issues and produce more successful outcomes for dynamic business processes.

\section{Visual Analytics Framework}

Visual analytics is the science of analytic reasoning facilitated by interactive visual interfaces [9]. It is meant to support exploratory analysis leading to discoveries since it is frequently applied to complex real world problems with large amounts of data. These problems are often openended with no clear path to solutions [9], and, as a result, it is often unclear what pertinent knowledge the data may contain [9]. Thus the analysis needs to be exploratory to support discovery of hidden relations, patterns, and trends. 


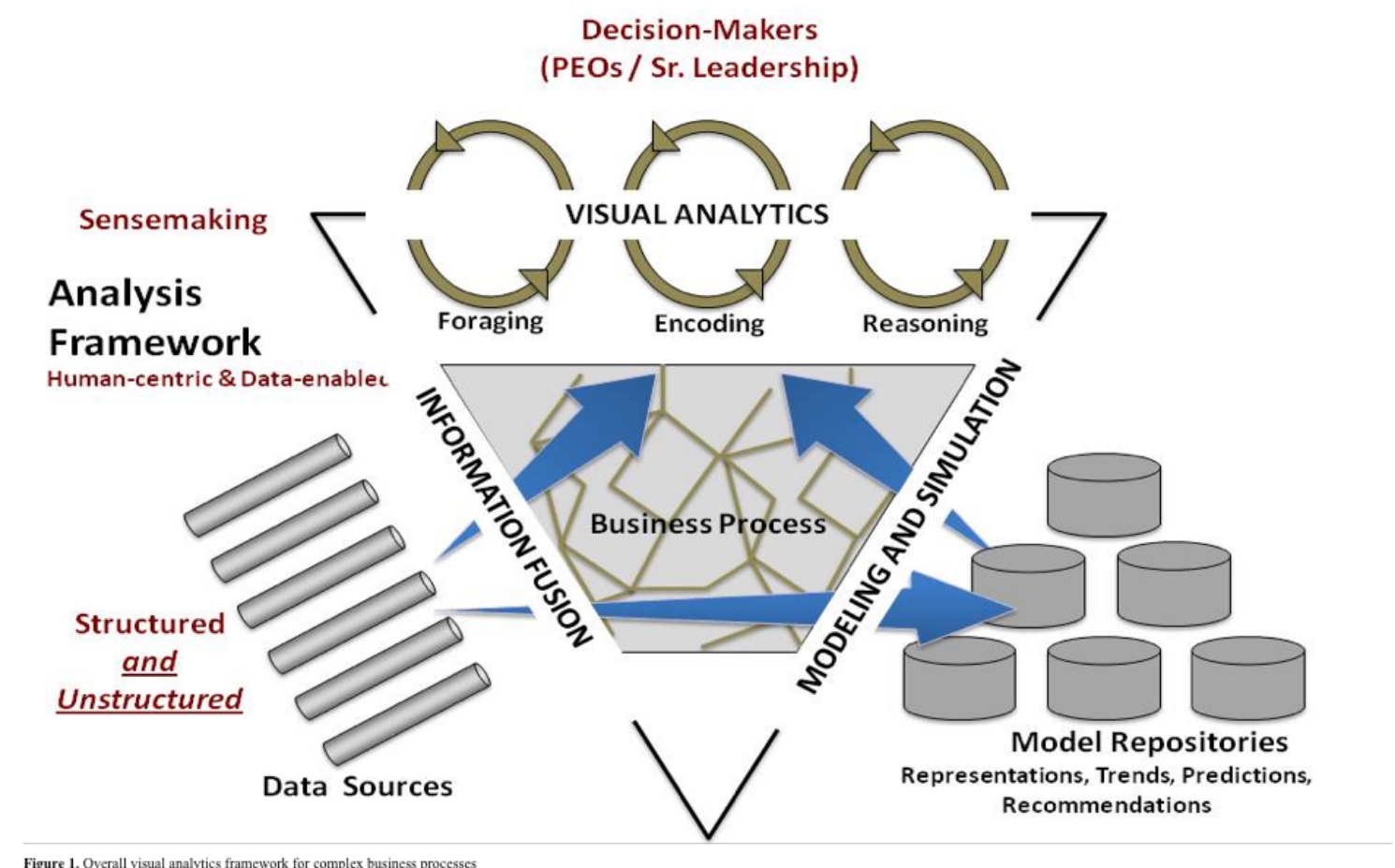

Figure 1. Overall visual analytics framework for complex business processes

Figure 2. Overall visual analytics framework for complex business processes.

Once discovered, these aspects should be investigated in detail, by referring back to original data and gathering additional evidence to confirm or refute hypotheses that are formed. A comprehensive visual analytics approach would support all these aspects. In this paper we apply such a comprehensive approach, employing visual analytics methods for both automated analyses and user-led exploration through the interactive visual interface.

Our first step is to embed the dashboard system in a visual analytics framework. This is depicted schematically in Figure 2. The repository of data sources (lower left) will inevitably contain both structured and unstructured data. Since a range of sources will be involved, the repository will be heterogeneous. Since useful information could be found anywhere (including perhaps outside the business) and, once found, should be used, some of these sources could be incomplete or fragmentary. Analytic tools must thus be flexible, able to handle different kinds of data, including incomplete data, yet producing results that contribute to a "common picture" that will be meaningful to the user.

Inevitably some of the data in a business process will be textual, containing comments, annotations, descriptions, reports, human communications, and so on. These texts will tend to be partly or mostly unstructured. Therefore we apply methods we have developed for extracting topics and topic-based events over time from unstructured texts [9].
These results are combined with named entity extraction for people's names, dates including future dates, and locations (if desired). The analysis can be applied, with approximations, either to streaming data [9] with results in a couple of minutes or less, or more slowly and accurately to histories over a period of time. These methods are quite flexible and have been applied to many different types of texts including reports, research papers, patent data, twitter streams, online news, and customer messages $[15,19,18]$. To these textual analyses, we add methods for exploring categorical data and numerical data, including finding trends over time. The categorical methods, for example, reveal relationships between multiple categories at selected times or time ranges, which are related to specific events in the topical event analysis. For all these methods plus the embedded dashboard, we develop a new interactive visual interface, as described in Sec. 6.

The analytic methods apply not only to the data repository but also to the business processes embedded in the middle of Figure 2 and, indeed, even to the model repository at the lower right. The business processes produce new information themselves and thus must be included to produce a most useful and comprehensive common picture. (This is indicated by thickening red lines that cross the middle of the figure.) In fact, as we have seen above, it is quite important to analyze information created during the business process since that will produce direct knowledge of the ca- 
pabilities and capacities of different parts of the process as well as signal when things are going wrong and what parts of the process are affected.

A general definition of a physical event is "a meaningful occurrence in space and time" [14]. For the purposes of this framework, we modify this definition to focus on "process events", which we define as "meaningful occurrences in time with respect to the process". In both cases, time is central. For this framework, bursts of activity over a relatively short time scale, unexpected trends or relationships (that appear in a short time span), or outliers could all qualify as process events. We have shown that events can be automatically found and organized (including putting them in a hierarchical structure) for social media such as Twitter [16]. This work, involving topic modeling, also demonstrates how users can quickly attach meanings to the events. The hierarchical structure becomes significant when one deals with complex data for which one can have many events. As data grows in size and comprehensiveness, it will tend to get complex in this way. Certainly the complex manufacturing and production processes considered here will benefit from hierarchical structuring, which will give them high level meaning and make the overall processes easier to understand.

\section{Virtual Sensors}

We now have the mechanisms we need to build the effective framework illustrated in Figure 2. The main idea is to instrument the whole business process (acquisition, production, deployment, etc.) with "virtual sensors". These sensors are flexible so that they can access any data stream (e.g., unstructured text, categorical data, numerical data, model outputs) anywhere, such as in the data repository, in the business processes themselves, or in the models. Typically sensors are placed at a set of general points that are effective for monitoring many different types of production. Then they are placed at additional points that are useful for a specific type of business process. Finally they can quickly be placed at other points by the manager, often to understand some abnormality in the process. This set of networked sensors then report to the visual analytics layer at the top of Figure 2. This layer provides a set of "sensemaking" capabilities to explore, understand, reason with, and test out hypotheses w.r.t. the sensor results. Since these results are expressed in a common language of process events, they can be displayed together and manipulated in an interactive visualization, though some details with respect to the events will be different depending on the underlying data or process. At any point, the manager can select one or more events and get at the underlying data.

The structure of the virtual sensor is depicted in Figure 3. The bottom layer grabs data directly from the business pro-

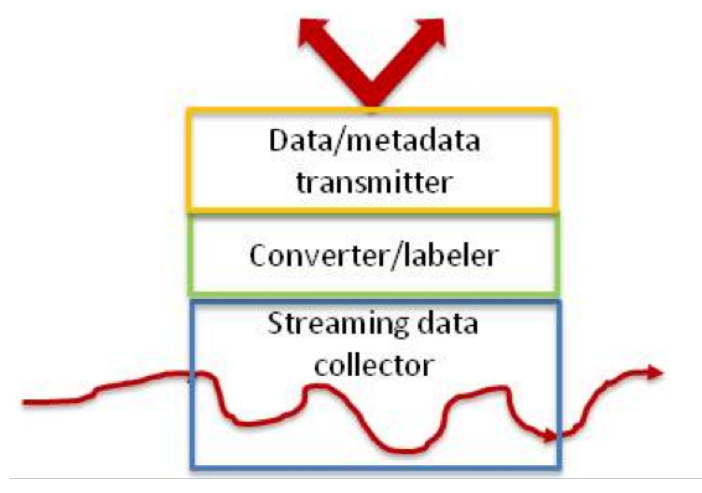

Figure 3. Virtual sensor structure.

cess stream. In the case of the business partner mentioned above, the dashboard already collection points that can be used to insert the sensors at several places. The middle layer converts these data to a standard internal format recognized by the analysis framework in Figure 2. It also attaches whatever metadata or labels are available (time range and time stamps, data descriptors, data units, etc.). The top layer transmits the data and metadata to the analysis framework.

Full analysis is performed by an analytics engine in the analysis framework, depending on the type of data. For instance, the analytics engine applies topic modeling and topic-based event extraction to unstructured text messages [19]. It applies temporal signal processing and anomaly detection to streams of numerical data to pull out both events and anomalies. It performs categorical and multidimensional analysis to categorical or attribute data. It performs relational and other statistical analyses to connect events across different types of data and to find similar signatures. These analyses are automated and all results are in a common event-based format regardless of the original input format (using the process event definition in Sec. 4.). Thus, for example, alignments of events from the text messages with events from categorical or numerical data streams would be readily spotted in the embedded visual interface described in the next section and then delved for deeper relationships. The common event-based format has the capacity for depicting additional "decorations" of the events stemming from details of the different types of original streaming data.

\section{The Embedded Visual Interface}

The visual interface is designed to support sensemaking in the business processes. More specifically, the visual interface incorporates 4 different views that highlight temporal, geospatial, and topical summaries using both the information available a priori (such as knowledge of the organization hierarchy) and information collected in real time by 


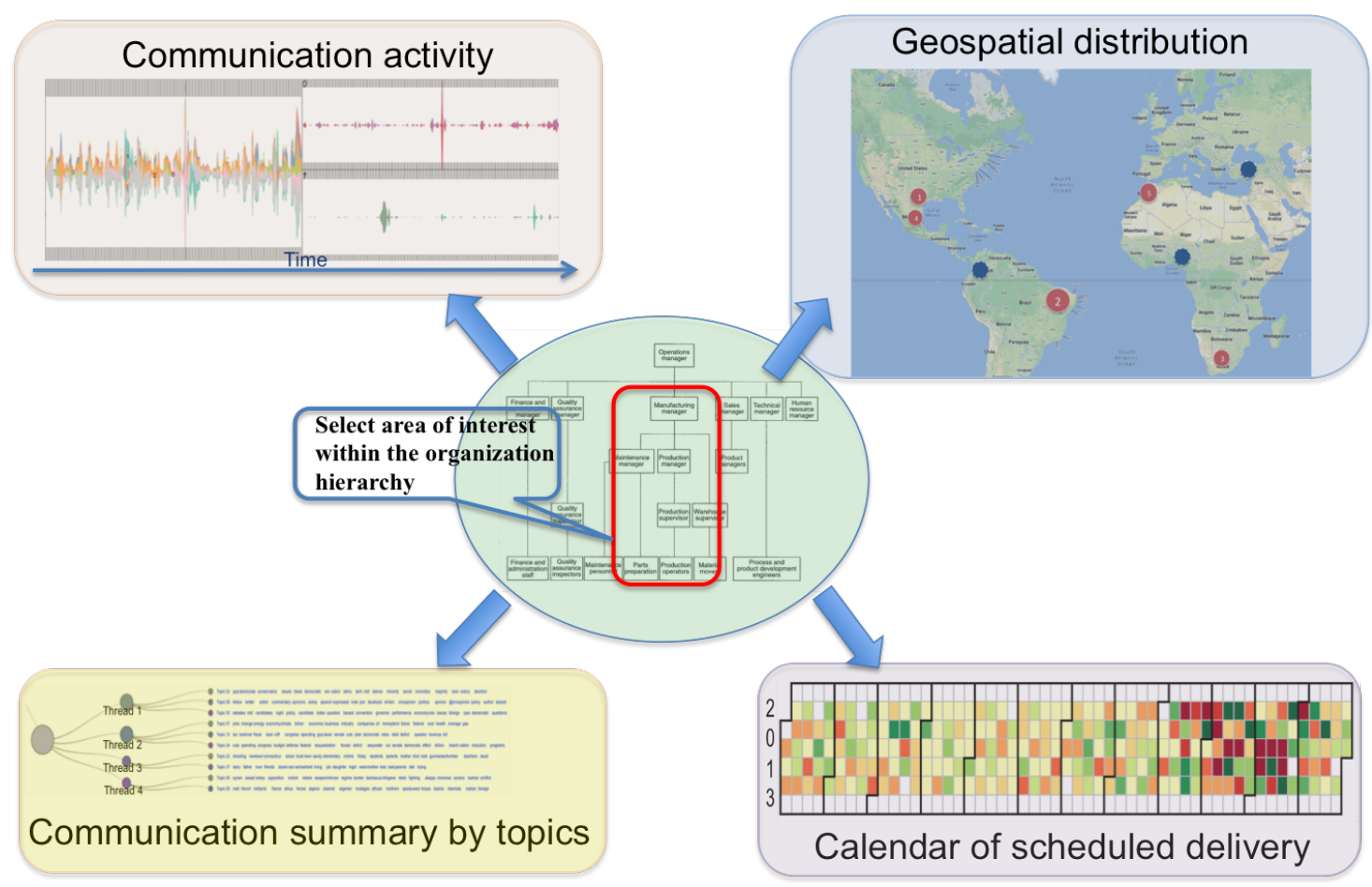

Figure 4. Embedded visual interface with labels to indicate functions.

the virtual sensors.

At the heart of the visual interface is the embedding into the external automatic and real-time sensory data repository and analyzer. The visual interface is set up to run on a single user's computer with intimate access to the external repository; it captures information flows to and from that user around office documents, calendars, emails, organizational charts, and Web pages, etc. Depending on the user analysis needs, the visual interface utilizes various meta-information that comes along with the virtual sensor results. In particular, it collects information about which documents, emails, and web pages were read for that user, and how long they were open. It also collects document metadata, such as information about the senders and recipients of email messages.

All this heterogeneous data is stored in a unified NoSQL external data repository for future analysis. On the fact collection level, tools like UpLib [8], can be utilized to extract information from and about each data input, including its title and authors, its text, the people and other entities that it mentions, its paragraphs and its images. All of the captured information is indexed and grouped with its related documents. On the event level, our signal processing then performs event analysis, time series analysis, clustering, and narrative reconstruction based on the collected organizational facts. All this is then interactively presented to the user through the embedded visualization interface.

\subsection{Visual Components}

Instead of presenting the diverse analyzed event structures through a keyword search interface, our framework embeds the investigative retrieval cues (e.g., who, when, where, what) into a coordinated multilevel visualization system.

At a high level, our visual interface encodes the four cues with a set of four visualizations, each of which presents a particular aspect of the organizational and business process activity. To provide a lower level detailed view, our interface also presents a visualization that integrates related activity information for a single worker or a sensor. Using this multi-level structure, the interface helps users to cohesively find the specific details they need.

Organizational Hierarchy View To give an overview of the complex operational environment, we designed an Organizational Hierarchy view that gives a cohesive overview while encapsulating organizational knowledge. Therefore, a user can start the analysis process by recalling event activities beginning from any retrieval cue that they remember (e.g., the department that mostly likely to initiated the process), or they can focus on events or cross-organization communication links that the automated analysis has identified as important. (See the application example in Sec. 7 for an illustration of the latter.) Interaction in any view cause 
updates in coordinated views so that the maximum associated information is provided permitting the user to converge on the desired conclusion quickly.

The Where: Geospatial view In the geospatial view, locations involved in the overall business process are highlighted in an interactive map. The importance of the location is represented by the size of the circles and could be determined by user-selected criteria. The numbering on the circle indicates the sequence of the manufacturing or delivering process. The color of the circles is used to denote main/alternative supply or manufacturing locations. The map supports standard user interactions such as zooming and panning. The map is coordinated with other views in that selections within the map will filter to events related to the chosen locations.

The When: Activity Heatmap View To facilitate multiscale temporal analysis, we developed an Activity Heatmap view that allows analysts to monitor events in both retrospective and future time frames. This view shows how a business process unfolds over time. It presents both the number of organizational activities (e.g. message exchange) that have interacted with business process, and the types of that business and its key personnel. This view is created as an interactive calendar for ease of interaction and it shows the temporal trends and patterns of organizational activities. Each cell in Figure 4C represents aggregated mentions of that date in all the existing business processes; dates that have been mentioned more frequently appear in a darker shade of blue. For example, we can quickly see that there are multiple delivery events that happen on the July 30th of this year.

Using this view, a manager can highlight a time range to select a subset of data (e.g., an hour to a day or months) to be analyzed retrospectively. Besides showing general trends and patterns, the temporal view also allows the user to drill down into time periods. When the user selects a time period on the horizontal axis in the center of the view, our visual interface will zoom into that period of time and present the most relevant business information.

The Who: Communication Activities between participating parties To help corporate managers and decision makers efficiently retrieve specific events of interest, we designed the Communication activities view to aggregate both the documents and the people that a user has interacted with during a particular period of time. Like Lee et al. [10], our activities view allows the user to filter and sort information based on automatically-extracted data facets, including different communication types and format. The user can visually depict the relationships between the extracted organizational facts with selected business projects. For example, the user can choose to see or hide activities with email, with office documents, with Web pages, or with people. Each of these facets can be turned on or off by pressing an associated button.

In order to fit the activity information into a reasonable guided exploration, our visual interface sorts events by importance, and displays the most important documents at the top and with the most salient presentation by computing the importance value centered around the relevance to its core business process. As described in Sec. ??, the organizational hierarchy view is also important in highlighting which events should be followed. In addition, to enable fast exploration, a summarized information panel (see Figure 4(D) right) is shown when the mouse hovers over a visual element representing an activity. Like the Document Card [17], this panel includes a readable thumbnail and aggregated information about that visual element. If the user needs more details, the user can double click on the visual element to bring up a specific detail view.

The What: Communication Summary by Topics To support the analysis of communications among different teams and parties during the overall business process, we designed the Communication Summary view, which provides an overview and permits analysis based on streaming or accumulated communication data. The analysis enables users to discover pressing issues that need to be addressed in order to keep the entire business process on track.

We assume most of the communication data are unstructured texts sent between communicating parties or attached as annotation to the stages of the business process. The virtual sensors collect all communications and put all the received communications into a database. The attribute in the database contains sender, receiver, time stamp, communication content, and other available information. All collected texts are then summarized into semantically meaningful topics using state-of-the-art topic models [2] as shown in Figure 4B. The topic summary presents individual topics to summarize what the communications are about. Coupling with the temporal trend of the topics shown in Figure 4A, one can quickly discover when a burst of communications has occurred and what issues were discussed. Other forms of data that are collected and analyzed are discussed in Sec. 7.

We now have complete support for the sensemaking process depicted at the top of Figure 2. The foraging stage is supported by the distribution of virtual sensors and their outputs. The encoding is supported by the conversion to a common event-based format in the analytics engine. The reasoning (including hypothesis-building and testing) is supported by the exploratory visual interface, which includes placement of virtual sensors where needed, and review of original data. All these stages are iterative and inter- 
connected through the visual interface. In the next section we will discuss how this sensemaking can be applied.

\section{An Application Example}

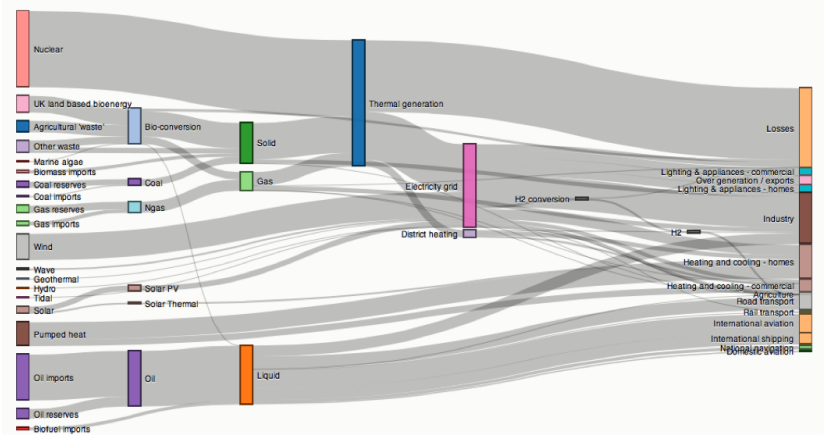

\section{Figure 5. Illustration of virtual sensors de- ployed in manufacturing process. Four vir- tual sensors are labeled V.S. in the diagram.}

In this section, we will build on the example given in the Introduction and illustrated in Figure 1. A real world manufacturing operation of any size will have a bigger organizational structure than depicted in Figure 1 and will have a layer of senior management (C-level: CEO, CIO, $\mathrm{CTO}, \mathrm{CFO}$, etc.).above the production manager. Because of complexity, the changing nature of dynamic JIT manufacturing and production, and the fact that signals of interest can come from unexpected places, our approach must be exploratory, bringing information from disparate sources into a common picture for evaluation and action. However, it is also data-driven, and since the initial data analytics especially are automated, there will be some tension between automated and user-directed processes. The visual analytics framework and the interactive visual interface are designed to minimize this tension and to make these processes work together in support of effective user actions.

A main aspect of the data-driven analysis is to identify process events (defined in Sec. 4) and to arrange them so that they tell a meaningful story when interpreted by the user. In the case of the dashboard used by our business partner, process events can be derived from numerical and categorical data collected at each stage of the dynamic manufacturing process, status reports indicating whether each stage or component of the process is on, behind, or ahead of schedule at that point, and individual annotation including comments by managers or engineers for that stage. There is also a mechanism for communication among managers, engineers, operators, and supervisors across the organization. The dashboard thus provides comprehensive data that we use in the above implementation of the visual analytics framework. However, though it provides comprehensive data, we have heard from the executive in charge that the dashboard is not sufficient to fulfill the need for on-time dynamic processing with minimum disruptiontoo many real or potentials points for breakdown can be overlooked; likewise too many opportunities for improvement and efficiency can be missed.

Thus we instrument the dashboard data and annotation collection points throughout the manufacturing process with virtual sensors, as illustrated in Figure 5. Process events are then identified in multiple ways. One way of doing so is by identifying bursts of activity over relatively short time scales in the topics derived from the textual annotations and communications. There could also be a trend in the numerical data that is unexpected compared to the normal manufacturing process. In the example here of not having a particular part when needed farther along in the manufacturing process, the burst of activity centers around one or more topics derived from both the annotations and the communications among workers indicating the part, the component system it goes into, and the supplier. An appropriate scale for this burst of activity is a few days. (In other words, text messages on the same topic accumulate in a burst over a few days.) Named entity extraction is also applied to the texts to indicate what sections of the organization (and individuals) are communicating, the names of parts, systems, and suppliers, and dates mentioned in the texts (either past or future). In addition, analysis of the inventory signal can indicate a drop in the supply of the crucial part over time that becomes significant enough to generate another event. The importance of the process events from the topic analysis is further raised because communication occurs across organizational boundaries between the parts manager, the quality assurance manager, and the technical manager (and their teams). The organizational knowledge of what is and is not unusual communication across the organizational hierarchy is encoded in the visual analytics framework. We have shown that such temporal bursts of topical activity can be automatically identified and that they can be connected to real events with high probability. We have successfully done this event analysis for streaming Twitter data (e.g., events during the development of the Occupy Wall Street movement over a year's time) [5] and collections of research proposal abstracts and full-text research papers [6] where the events are often connected with new programs started by funding agencies. These quite different text collections and quite different time scales demonstrate that the topical event-identification methods are general.

Up to this point the analysis is all automatic, but the process event results based on both statistical techniques (e.g., signal processing) and process and organization knowledge permits highlighting of events, anywhere in the process or 
organizational structure, that are likely to be of interest to the high-level manager. The manager starts to interact with these results via the visual interface in Figure 4. Through the central organization hierarchy view, he sees highlighted links between the parts group and the quality manager and technical management groups that he knows are unusual. A check of the communication activity view shows when these bursts of communication occurred. The problem has not yet shown up in the calendar view (i.e., the affected process components are not yet falling behind schedule). He can then dig down to the actual texts of topical messages and can filter for geographical distributions of both suppliers and the finished systems, which will be displayed in the geographical view. He can set a new virtual sensor to watch the inventory stream (and to collect historical data). If there is a more complex problem, there may be several relevant topics. In this case, the hierarchical topic view (communication summary view) is useful. The view shows which topics are related and can be grouped and also permits regrouping by the manager. The data associated with the topics, including text messages and other data, are reorganized as well. In summary, the manager can quickly find issues that should concern him and then effectively analyze them, moving towards making key decisions.

The visual analytics framework has other useful attributes. The process of investigation by the manager that leads to a conclusion and a plan of action is itself captured in terms of selected process events, topics, the manager's own annotations, and interactions with the views in the visual interface. We have found in our studies of bank fraud analysts that an interface such as this can reveal high level strategies and its own meaningful story of how an expert reaches a decision [4]. This story can be shared with other managers, giving a rich argument for a course of action. In addition, like the fraud analysts, the managers in this dynamic manufacturing process have expertise developed through experience that is not easy to share. However in the case of our partner, the problem is exacerbated because, due to the nature of the business, there is some flow of managers into and out of their positions and thus a knowledge and experience gap. Use of the visual interface and underlying analytics by experts for a variety of situations can be captured and made available as training modules for these new managers, giving them insights that would be hard to obtain without long experience.

\section{Conclusion}

We have presented a visual analytics framework for handling complex business processes. It is applied to dynamic just in time manufacturing, but it is applicable to a range of agile business processes.

In order to organize data that can come from anywhere in the complex business process and can be in a variety of types, we have introduced the concepts of virtual sensors and process events. The virtual sensors permit collection from the streams of data at any point in the process and transmission of the data in a form ready to be analyzed by the central analytics engine. Process events provide a uniform expression of data of different types in a form that can be automatically prioritized and that is readily meaningful to the users. Through the visual interface, the user can place the virtual sensors, interact with and group the process events, and delve into the details of the process at any point. The visual interface provides a multiview investigative environment for sensemaking and decisive action by the user.

We have shown that with this visual analytics environment, the user can answer the major questions that must be addressed in order to achieve a successful dynamic production environment. These include finding overall trends and then detailed specific trends in the production process; determining, as early as possible, what may be going wrong and where in the process; determining what the capacities of various parts of the process are and what resource reallocation is needed; and determining what expertise should be deployed to most quickly solve a problem.

We are now working on detailed set up and evaluation of this framework. The response to the initial design and implementation by our partner is quite positive. The eventand process-based analysis presented here is of particular interest because it will bring data from multiple streams (especially textual annotations and comments) into a common view for analysis and decision-making. These are new capabilities for the partner. We are now embarking on a full project to use these capabilities in detail. This will lead to improvements and to the reporting of concrete case studies.

\section{References}

[1] B. Azvine, D. D. Nauck, C. Ho, K. Broszat, and J. Lim. Intelligent process analytics for crm. BT Technology Journal, 24(1):60-69, Jan. 2006.

[2] D. M. Blei, A. Y. Ng, and M. I. Jordan. Latent dirichlet allocation. J. Mach. Learn. Res., 3:993-1022, Mar. 2003.

[3] R. Bobrik, M. Reichert, and T. Bauer. View-based process visualization. In Proceedings of the 5th international conference on Business process management, BPM'07, pages 88-95, Berlin, Heidelberg, 2007. Springer-Verlag.

[4] R. Chang, M. Ghoniem, R. Kosara, W. Ribarsky, J. Yang, E. Suma, C. Ziemkiewicz, D. Kern, and A. Sudjianto. Wirevis: Visualization of categorical, time-varying data from financial transactions. In Proceedings of the 2007 IEEE Symposium on Visual Analytics Science and Technology, VAST '07, pages 155-162, Washington, DC, USA, 2007. IEEE Computer Society.

[5] W. Dou, D. Wang, Z. Ma, and W. Ribarsky. Discover diamonds-in-the-rough using interactive visual analytics 
system-tweets as a collective diary of the occupy movement, 2013.

[6] W. Dou, X. Wang, R. Chang, and W. Ribarsky. Paralleltopics: A probabilistic approach to exploring document collections. In Visual Analytics Science and Technology (VAST), 2011 IEEE Conference on, pages 231-240, 2011.

[7] G. Hansen. Automated Business Process Reengineering: Using the Power of Visual Simulation Strategies to Improve Performance and Profit. Prentice-Hall, Englewood Cliffs, 1997.

[8] W. C. Janssen. The uplib personal digital library system. In Proceedings of the 5th ACM/IEEE-CS joint conference on Digital libraries, JCDL '05, pages 410-410, New York, NY, USA, 2005. ACM.

[9] T. Kraft, X. Wang, J. Delawder, D. W., L. Yu, and W. Ribarsky. Less after the fact: Investigative visual analysis of events from streaming twitter. In IEEE symposium on large data analysis and visualization, 2013.

[10] B. Lee, G. Smith, G. G. Robertson, M. Czerwinski, and D. S. Tan. Facetlens: exposing trends and relationships to support sensemaking within faceted datasets. In Proceedings of the SIGCHI Conference on Human Factors in Computing Systems, CHI '09, pages 1293-1302, New York, NY, USA, 2009. ACM.

[11] A. Leoni, M. de, W. van der, and A. t. Hofstede. Process mining and visual analytics: Breathing life into business process models. BPM Center Report BPM-11-16, 31pp, 2011.

[12] M. Leoni, W. Aalst, and A. Hofstede. Visual support for work assignment in process-aware information systems. In M. Dumas, M. Reichert, and M.-C. Shan, editors, Business Process Management, volume 5240 of Lecture Notes in Computer Science, pages 67-83. Springer Berlin Heidelberg, 2008.

[13] P. Oude Luttighuis, M. Lankhorst, R. Van De Wetering, R. Bal, and H. Van Den Berg. Visualising business processes. Comput. Lang., 27(1-3):39-59, Apr. 2001.

[14] W. Ribarsky, E. Sauda, J. Balmer, and Z. Wartell. The whole story: Building the computer history of a place. In Hawaii International Conference on Systems Science (HICSS 2012, 2012.

[15] S. A. Ruffa. Going lean: How the best companies apply lean manufacturing principles to shatter uncertainty, drive innovation, and maximize profits. AMACOM (American Management Association), 2008.

[16] A. Streit, B. Pham, and R. Brown. Visualization support for managing large business process specifications. In W. Aalst, B. Benatallah, F. Casati, and F. Curbera, editors, Business Process Management, volume 3649 of Lecture Notes in Computer Science, pages 205-219. Springer Berlin Heidelberg, 2005.

[17] J. Teevan, C. Alvarado, M. S. Ackerman, and D. R. Karger. The perfect search engine is not enough: a study of orienteering behavior in directed search. In Proceedings of the SIGCHI Conference on Human Factors in Computing Systems, CHI '04, pages 415-422, New York, NY, USA, 2004. ACM.
[18] X. Wang, W. Dou, Z. Ma, J. Villalobos, Y. Chen, T. Kraft, and W. Ribarsky. I-si: Scalable architecture for analyzing latent topical-level information from social media data. Comp. Graph. Forum, 31(3pt4):1275-1284, June 2012.

[19] X. Wang, W. Dou, W. Ribarsky, D. Skau, and M. X. Zhou. Leadline: Interactive visual analysis of text data through event identification and exploration. In Proceedings of the 2012 IEEE Conference on Visual Analytics Science and Technology (VAST), VAST'12, pages 93-102, Washington, DC, USA, 2012. IEEE Computer Society.

[20] W. Wright. Business visualization adds value. Computer Graphics and Applications, IEEE, 18(4):39-, 1998. 